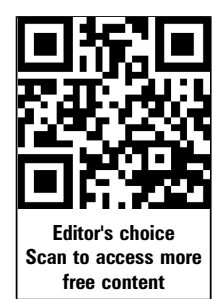

- Additional material is published online only. To view please visit the journal online (http://dx.doi.org/10.1136/ postgradmedj-2014-132847).

${ }^{1}$ Royal Prince Alfred Hospital, Camperdown, New South Wales, Australia ${ }^{2}$ Sydney Medical School, University of Sydney, Sydney, New South Wales, Australia ${ }^{3}$ Health Education and Training Institute, Gladesville, New South Wales, Australia ${ }^{4}$ Sydney School of Public Health/Faculty of Medicine, University of Sydney, Sydney, New South Wales, Australia

\section{Correspondence to} Dr Nishmi Gunasingam, Medical Training and Administration Unit, Royal Prince Alfred Hospital, Missenden Road, Camperdown, NSW 2050, Australia; nishmi@gmail.com

Received 15 July 2014 Revised 22 January 2015 Accepted 16 February 2015 Published Online First 9 March 2015

\title{
Reducing stress and burnout in junior doctors: the impact of debriefing sessions
}

\author{
Nishmi Gunasingam, ${ }^{1,2}$ Kharis Burns, ${ }^{1,2}$ James Edwards, ${ }^{1,2,3}$ Michael Dinh, ${ }^{1,2}$ \\ Merrilyn Walton ${ }^{4}$
}

\begin{abstract}
Background Internship and residency are difficult times with novice practitioners facing new challenges and stressors. Junior doctors may experience burnout, a syndrome that encompasses three dimensions: emotional exhaustion, depersonalisation and reduced personal accomplishment. While there is some existing literature on the prevalence of burnout in junior doctors, there are few studies on interventional strategies.
\end{abstract}

Aims This study aimed to examine the prevalence of burnout in a cohort of junior doctors and whether debriefing sessions reduced levels of burnout.

Methods A prospective randomised controlled study of a convenience sample of postgraduate year 1 doctors in a single hospital was undertaken during a rotation term in 2011. All participants completed a questionnaire using a validated tool, the Maslach Burnout Inventory, to determine the prevalence of burnout. They were then randomly assigned to a group who were to receive four debriefing sessions over 2 months, or, to the control group, who had no debriefing sessions. Quantitative and qualitative analyses were conducted.

Results Thirty-one postgraduate year 1 doctors participated in the study, with 13 being assigned to the group receiving debriefing sessions and 18 assigned to the control group. At baseline, 21/31 (68\%) participants displayed evidence of burnout in at least one domain as measured by the Maslach Burnout Inventory. Burnout was significantly higher in women. There was no significant difference in burnout scores with debriefing. The intervention was well received with 11/18 (61\%) suggesting they would recommend the strategy to future junior doctors and 16/18 (89\%) found that the sessions were a source of emotional and social support.

Conclusions Burnout is prevalent among postgraduate year 1 doctors, and they value the emotional and social support from attending debriefing sessions. A larger study is required to determine if debriefing can reduce the incidence of burnout in junior doctors.

\section{INTRODUCTION}

Many medical graduates feel unprepared for clinical practice when confronted with the realities of the wide breadth of duties that the job entails. They are required to manage acutely ill patients and stressful workloads. It is not surprising, therefore, that some of them will suffer psychological stress that reduces their functioning, experience increased psychiatric morbidity along with job and life dissatisfaction. Doctors who are emotionally exhausted, cynical about the importance or value of one's occupation or show diminished interest in their work are experiencing features of burnout. ${ }^{1-4}$
Burnout encompasses three dimensions; emotional exhaustion, depersonalisation and reduced personal accomplishment. ${ }^{5}$ It is a response to chronic emotional and interpersonal stressors and has been known to affect professions requiring significant and intense involvement with people, such as doctors and nurses. ${ }^{3}$

The consequences of burnout to the individual are significant. Personal health is often neglected, with one study indicating that $65 \%$ of medical practitioners felt unable to take time off work when ill, and 92\% had self-prescribed medication. ${ }^{4}{ }^{6}$ Burnout, depression and sleep deprivation have also been shown to significantly increase the odds of a motor vehicle accident. ${ }^{7}$

Medical staff are not the only ones affected; patient care can also be impeded. Burnout has been linked to deferred clinical decision making and increased perceived medical errors. ${ }^{5}$ It has long been recognised that stress levels affect intellect, judgement and technical ability. Fatigue, distress and depression have been separately shown to be associated with medical errors. Addressing burnout is critical, as it not only has an impact on the quality of life of junior doctors, but also patient safety and health outcomes. ${ }^{8} 9$

Burnout is common in junior doctors. It has been reported to occur in $18-82 \%$ of junior doctors and increases towards the end of the intern year. ${ }^{3}$ One Australian study on junior doctor wellbeing found that $71 \%$ of doctors were concerned about their own health and the majority had low job satisfaction (71\%), burnout (69\%) and compassion fatigue $(54 \%) .^{2} 10$

There have been few studies investigating strategies to prevent or reduce burnout in junior doctors in comparison with the literature on its prevalence. Previous interventions have aimed to implement change at either the organisational or individual level. ${ }^{11}$

Long working hours, poor work-life balance and the inability to commit to personal and social activities outside of work has been associated with burnout. ${ }^{2}$ However, there is conflicting opinion on the implementation of work hour limits at an organisational level reducing the prevalence of burnout.

The work of a junior doctor is inherently stressful. High levels of responsibility coupled with role ambiguity and low levels of decision latitude are common and are associated with an increased risk of burnout. ${ }^{12}$ The developing pressures associated with job prospects and career trajectories also contribute to stress on the junior doctor. Given that it 
is unlikely that these factors change, most strategies to reduce burnout have concentrated on modifying individuals' response to work stress.

Approaches that have addressed stress reduction in junior doctors' work have included educational workshops on recognising signs of stress. ${ }^{13} \mathrm{~A} 9$-weekly team training session, led by a psychotherapist, for resident physicians and nurses, focused on improved communication and role conflict, and was well received by its participants. ${ }^{14}$ Meditation based techniques have also been effective. Shapiro demonstrated that a meditation based stress reduction intervention reduced anxiety and depression, and increased empathy levels in medical students. ${ }^{15}$ Another form of meditation (using a breathing technique) has also been shown to improve emotional exhaustion levels in family physicians. ${ }^{16}$ However, these methods do not address each of the dimensions of burnout or the underlying contributory chronic emotional and interpersonal stressors.

The establishment of debriefing strategies to reduce burnout in junior doctors has been recommended with varying success. $^{2}{ }^{4} 1718$ Debriefing, in the context of chronic workrelated emotional and interpersonal stressors, can be described as an opportunity to meet with peers and have a facilitated discussion with a senior and trusted health professional. It essentially involves peer support, feedback, mentoring and problem solving. Increased support and feedback has been shown to reduce work-related psychological stress. ${ }^{19}$ Sharing experiences is a possible antidote to burnout, with peer -support groups helpful in prevention. ${ }^{20-22}$ However, the efficacy of debriefing to reduce burnout in junior doctors has not been evaluated.

The objective of the study is to determine the prevalence of burnout in a cohort of junior doctors and to evaluate the effectiveness of debriefing sessions in reducing burnout.

\section{METHODS}

Design

This study was a randomised controlled trial using quantitative and qualitative methods to understand the multiple perspectives and contextual factors associated with burnout.

\section{Participants and setting}

A convenience sample of postgraduate year 1 doctors in a metropolitan teaching hospital was invited to participate through an information session about the study and distribution of participant information leaflets. The study was conducted during the third term of a five-term year in 2011.

\section{Inclusion and exclusion criteria}

The sample of interns invited to participate were those who were based at the teaching hospital during term 3 of 2011. Interns who were seconded to other hospitals were excluded.

\section{Data collection}

Phase 1

The prevalence of burnout was measured by asking each participant, at the start of the term, to complete the Maslach Burnout Inventory (MBI). The MBI, a validated tool, is a questionnaire comprising the following three domains: emotional exhaustion, cynicism and professional efficacy. These domains are addressed individually and as a composite score, calculated by summing the cynicism and exhaustion scales and then subtracting the professional efficacy score. The higher the score the higher the level of burnout with a negative burnout score indicating no burnout. When the domains are analysed individually, a high sub-score in emotional exhaustion or depersonalisation is considered
Table 1 Maslach Burnout Inventory scoring system ${ }^{23}$

\begin{tabular}{llll}
\hline \multirow{2}{*}{ Severity } & Categorisation & & \\
\cline { 2 - 4 } & Professional efficacy & Emotional exhaustion & Cynicism \\
\hline High & 30 or over & 16 or over & 13 or over \\
Moderate & $24-29$ & $8-15$ & $6-12$ \\
Low & $0-23$ & $0-7$ & $0-5$ \\
\hline
\end{tabular}

indicative of clinically significant burnout (table 1). ${ }^{23}{ }^{24}$ The MBI has become the gold standard for identifying burnout in the medical research literature, with individual and composite scores considered acceptable. ${ }^{25} 26$

\section{Phase 2}

The MBI was repeated by all participants at the completion of the term, approximately 2 weeks after the last debriefing session. The repeat MBI was done to assess for change in the burnout scores following the debriefing intervention.

\section{Phase 3}

Participants in the intervention arm completed a written evaluation survey assessing their opinions on the debriefing sessions (see online supplementary appendix 1 document).

\section{Phase 4}

A focus group of six participants, sourced from the intervention arm based on availability (all participants in intervention arm were invited), was also conducted after the debriefing sessions. The focus group, led independently by a trained facilitator, explored themes around work-related stressors, coping mechanisms and potential strategies to improve Junior Medical officer well-being. Examples of questions explored in the focus group are presented in box 1 . The session was recorded and converted to an audio file that was then electronically transcribed for thematic analysis.

The results from the three methods (MBI, evaluation survey and focus group) were then triangulated.

\section{Process}

Participants were allocated a unique identifying number to maintain anonymity. A computer generated randomisation code

\section{Box 1 Excerpt from focus group transcript questions}

1. Did anyone experience work-related stress during your internship?

2. Coping strategies for stress-what are the sort of things that you do in order to cope with stress related to your work?

3. Are there other things besides the time pressures or those particular immediate things at work that stress you out? Are there other bigger life things?

4. How about your work-life balance-are you finding that okay to manage? Do you have particular stresses in relation to that?

5. Can you suggest some structures that could help junior doctors manage these stressful situations in general not just after a particular stressful event? 
allocated participants to the debriefing intervention or control group.

The intervention group attended four debriefing sessions led by experienced senior health professionals who designed and followed a protocol on conducting the session. Lead investigators organised sessions fortnightly, for approximately $1 \mathrm{~h}$ after the working day, onsite at the hospital. These sessions were strictly confidential.

Preparation for debriefing involved planning the management of the sessions, discussing the recording sheet to identify themes raised by the participants and agreement for a process if a participant disclosed a serious matter warranting action. Each session started with the facilitator asking each participant how the previous fortnight had been and then allowing the topics for discussion to emerge generically from their concerns and experiences. Facilitators had topics of themes to raise if no participant had anything to say (box 2). Facilitators kept a log of the themes covered. The debriefing sessions were not recorded for privacy purposes and to encourage open discussion in a confidential environment.

\section{Data analysis}

Quantitative data were analysed using SAS V.9.3 (SAS Institute, Cary, North Carolina, USA). Descriptive statistics were used for demographic information. Mean scores were expressed with 95\% CIs. Multivariable linear regression was used to model preintervention scores adjusting for age, relationship status, gender and unrostered overtime. Repeated measures mixed models were used to compare preintervention and postintervention scores within each domain (physical exhaustion, cynicism, efficacy) and total burnout score (defined in this study as the personal efficacy minus the combined cynicism and exhaustion scores) to account for correlation between longitudinal data and missing values. Burnout was also categorised as positive if there were high levels of exhaustion or cynicism regardless of efficacy

\section{Box 2 Example guide to themes discussed in debriefing} sessions

1. Challenges associated with internship?

2. What common things do you worry about related to work?

3. Coping strategies to deal with job-related stress
A. Talking about it/Formal debriefing
B. Exercise
C. Alcohol
D. Other drugs
E. Recreational/relaxation activities
F. Spending time with family/friends
G. Discussing concerns with a mentor
H. Sleep
I. Prayer or meditation
J. Formal counselling or therapy
K. Change of employment

4. Work-life balance

5. Work relationships and level of support at work

6. Opinions on ways to better support junior doctors

7. Identifying issues contributing to job-related stress
A. Environmental factors
B. Personal factors
C. Job-specific factors

scores. Generalised estimating equations were then used to compare the difference in burnout categories in the control and intervention groups. The change over time in burnout scores or burnout categories was modelled using interaction variables between time of assessment (baseline and debriefing session) and the study group. $p$ Values reported in tables represent the significance values of the interaction variable with the debriefing sessions analysed thematically. Specifically, the electronically transcribed audio file was scanned for key theme analysis. A difference in three points on total burnout scores was assumed to represent a relevant effect of the debriefing intervention, although no previous studies have determined this. Assuming a SD of 10 points, a power of 0.8 , and a two-tailed $\alpha$ value of 0.05 , a sample size of 91 participants would be required for a full study. However, this was a pilot study to assess feasibility of planned debriefing sessions.

\section{RESULTS}

\section{Participants}

Thirty-one of 52 invited interns entered this study. Thirteen attended debriefing sessions (the intervention) and the 18 interns in the control group received no additional support. Non-participation was mainly due to the secondment to rural hospitals (14/52) (Consort Diagram, see online supplementary appendix 2 document). Demographic information is summarised in table 2 and a description of internship requirements, terms and the hospital surveyed is in table 3 .

\section{Prevalence of burnout}

At baseline 21/31, 68\% (95\% CI 52\% to 84\%) of participants displayed evidence of burnout in at least one domain as measured by the MBI scale. High levels of emotional exhaustion and cynicism were seen in $14 / 31(45 \%)$ and $17 / 31(55 \%)$ of participants, respectively. Low professional efficacy was reported in $5 / 31(16 \%)$ of the cohort at baseline.

In the poststudy group of the entire cohort, the level of burnout was $17 / 31$ (55\%, 95\% CI 37\% to 73\%). 11/31 (36\%) reported

Table 2 Demographic information

\begin{tabular}{lll}
\hline $\begin{array}{l}\text { Number of participants } \\
\text { Gender }\end{array}$ & $\begin{array}{l}\text { Intervention } \mathbf{n}(\%) \\
\mathbf{n}=13\end{array}$ & $\begin{array}{l}\text { Control } \mathbf{n}(\%) \\
\mathbf{n}=18\end{array}$ \\
$\begin{array}{lll}\text { Male } \\
\text { Age (years) }\end{array}$ & $8(62)$ & $8(44)$ \\
$20-24$ & $4(31)$ & $10(56)$ \\
$25-30$ & $9(69)$ & $6(33)$ \\
$>30$ & $0(0)$ & $2(11)$ \\
Rotation & & \\
Emergency & $2(15)$ & $3(17)$ \\
Medical & $6(46)$ & $6(33)$ \\
Surgical & $5(38)$ & $8(44)$ \\
Relief & $0(0)$ & $1(6)$ \\
Relationship status & & $8(44)$ \\
Single & $8(61)$ & $16(89)$ \\
Training location & & \\
Australia trained & $13(100)$ & $9(50)$ \\
Total hours worked per week & & $5(28)$ \\
$40-50$ & $3(23)$ & $4(22)$ \\
$50-60$ & $9(69)$ & \\
$60-70$ & $1(8)$ &
\end{tabular}


Table 3 Hospital descriptors, internship requirements and terms

$\begin{array}{ll}\begin{array}{l}\text { Hospital location } \\ \text { Number of hospital beds }\end{array} & \begin{array}{l}\text { Inner Sydney City } \\ \text { Number of postgraduate year } 1\end{array} \\ \begin{array}{l}\text { doctors in } 2011^{*} \\ \text { Number of terms completed in } \\ \text { internship }\end{array} & 52 \\ \text { Term requirements } & \begin{array}{l}1 \text { emergency term, } 1 \text { surgical term and } 1 \\ \text { medical term } \\ 80 \mathrm{~h} \text { (baseline) }\end{array} \\ \begin{array}{l}\text { Contracted working hours per } \\ \text { fortnight }\end{array} \\ \begin{array}{l}\text { *In every term a number of postgraduate year } 1 \text { doctors are seconded to peripheral } \\ \text { hospitals. This number reflects the total number recruited at the beginning of the } \\ \text { year. }\end{array}\end{array}$

feeling emotionally drained once a week to everyday, and 17/31 $(45 \%)$ reported they felt 'used up' at the end of the working day. The mean (SD) preintervention burnout scores were significantly higher in women compared with men $(+3.6(9.5)$ vs $-5.8(10.9)$, $\mathrm{p}=0.016$ ), respectively, with an estimate of the difference of -9.4 (95\% CI -17.0 to -1.9 ). Additionally, a higher proportion of women met criteria for burnout in comparison with men (13/15 $(87 \%)$ vs $8 / 16(50 \%), \mathrm{p}=0.029)$ (table 4).

The association between burnout score and gender remained significant after adjusting for age, unrostered hours and relationship status, with adjusted burnout scores for women on average 9.4 points higher than men $(95 \%$ CI 1.0 to $17.3, \mathrm{p}=0.027)$.

Mean burnout scores and subscale scores stratified by the debriefing intervention group and preintervention and postintervention periods are summarised in table 5. Using mixed modelling, the change in mean burnout scores was not different between the control and intervention groups (table 6). With respect to the individual domains there was no change in emotional exhaustion, cynicism or professional efficacy subscale scores in the intervention group and no change in the control group scores (table 5).

Identified stressors elicited during the focus groups included difficulty in maintaining work-life balance, lack of support by senior staff and uncertainties in relation to career paths and training (box 3). Identified coping mechanisms included socialising, alcohol, informal and formal debriefings with colleagues, and formal support systems from hospital teams (box 4).

Themes emerging from the debriefing sessions included managing staff relationships, inadequate communication and supervision. Inadequate supervision was a common stressor to both groups. In the debriefing group supervision was directly discussed in relation to specific cases, while in the focus group participants described lack of senior staff as a recognised stressor. It was also apparent from the debriefing sessions and focus group that neglecting personal health was not uncommon, supporting literature findings. Focus group and debriefing group members mentioned little time for exercise, or other social activities apart from weekends.
The evaluation survey found that the formal debriefing sessions were well received, with $6 / 10(60 \%)$ suggesting they would recommend this strategy to future junior doctors. Participants felt that it was 'good to hear peers having similar issues' and 9/10 (90\%) found the debriefing sessions to be a source of emotional and social support (box 5). The post hoc analysis also found that interns would welcome such sessions on a regular basis.

\section{DISCUSSION}

Our study found that more than half of the junior doctors surveyed displayed high levels of burnout but that debriefing sessions, while considered a valuable support mechanism, did not improve burnout scores using the MBI.

Female interns experienced higher levels of burnout, but no association was shown with age, current term or hours worked, confirming previous studies which also showed no causal relationship between burnout and demographic factors. ${ }^{27}$ Our findings confirm results of a larger-scale study where internal medical female residents demonstrated higher levels of emotional exhaustion and depersonalisation than men. ${ }^{28}$ Given our findings, factors associated with being female and the work environment, is worthy of further study.

Sources of stress focused on work-life balance, long working hours and continually adjusting to new environments and terms. These concepts, previously well documented, highlight the ongoing relevance of addressing these issues at an organisational level. The stressors identified largely reflect previous research findings, with lack of support from senior staff, and ambiguity of future career progression raised as common concerns.

Alcohol and informal debriefing among colleagues were commonly employed coping tools, supporting previous concerns raised about the use of alcohol and drugs by junior doctors to deal with stress in the literature. ${ }^{2}$ Informal debriefing with peers was seen by the debriefing group and focus group participants as a useful way to manage stress and prevent burnout. It appears that junior doctors have themselves identified and facilitated a strategy to manage the stress of internship. The difficulty with relying upon this as a strategy is that it only suits some of the cohort. Many have family responsibilities, do not drink alcohol and are not part of a 'social' network. Assuming the merits of debriefing it makes sense to provide a similar opportunity for all junior doctors in a safe and secure environment; one that hospitals should take responsibility for.

Despite no change in burnout scores using the MBI, the welcomed response and general acceptance support the potential for further application of debriefing sessions. Specifically, the comfort it provided to know that others were having similar struggles was a strong theme identified in this study and interns drew reassurance from this. This provided a sense of support and community and was identified as a source of relief.

Career uncertainty and the likelihood of more doctors pursuing fewer jobs may increase stress levels for interns with the workplace becoming more complex than ever. Older and sicker

Table 4 Maslach Burnout Inventory scores pre intervention and post intervention according to gender for the entire cohort

\begin{tabular}{|c|c|c|c|c|c|c|}
\hline & \multicolumn{3}{|c|}{ Preintervention mean $(95 \% \mathrm{Cl})$} & \multicolumn{3}{|c|}{ Postintervention mean $(95 \% \mathrm{Cl})$} \\
\hline & Emotional exhaustion & Cynicism & Professional efficacy & Emotional exhaustion & Cynicism & Professional efficacy \\
\hline Male & $10.4(7.2$ to 13.7$)$ & 11.9 (9.3 to 14.5$)$ & 28.1 (25.0 to 31.2$)$ & $11.5(7.5$ to 15.5$)$ & $11.3(7.6$ to 14.9$)$ & $28.4(25.0$ to 30.9$)$ \\
\hline Female & $17.7(14.5$ to 21.0$)$ & 13.7 (10.6 to 16.9 ) & 27.9 (24.9 to 30.8 ) & 16.2 (12.7 to 19.7$)$ & 14.6 (10.2 to 19.1$)$ & 29.4 (27.0 to 31.7$)$ \\
\hline
\end{tabular}


Table 5 Maslach Burnout Inventory scores pre intervention and post intervention for control versus intervention

\begin{tabular}{|c|c|c|c|c|c|c|}
\hline & \multicolumn{3}{|c|}{ Preintervention mean $(95 \% \mathrm{Cl})$} & \multicolumn{3}{|c|}{ Postintervention mean $(95 \% \mathrm{Cl})$} \\
\hline & Emotional exhaustion & Cynicism & Professional efficacy & Emotional exhaustion & Cynicism & Professional efficacy \\
\hline Control & 13.4 (10.4 to 16.5$)$ & 12.7 (10.0 to 15.3$)$ & 26.1 (23.0 to 29.1 ) & 13.5 (10.8 to 16.3$)$ & 12.9 (9.7 to 16.2$)$ & 27.9 (25.7 to 30.2$)$ \\
\hline Intervention & 14.7 (9.7 to 19.6$)$ & 12.9 (9.7 to 16.2$)$ & 30.7 (28.8 to 32.6$)$ & 14.2 (8.4 to 20.0$)$ & 12.8 (7.3 to 18.3$)$ & $30.2(27.6$ to -32.7$)$ \\
\hline
\end{tabular}

patients alone, with complex healthcare needs, create many opportunities for failure for the novice doctor. Burnout, therefore, is also an organisational responsibility as well as an individual one. Debriefing programmes are low cost, easily coordinated and potentially have the capacity to offer institutional support throughout the course of an internship. The availability of personnel trained in debriefing would be a valuable asset to any unit supervising training junior doctors. Burnout is not unique to doctors, and implementation of training in debriefing may benefit all healthcare staff involved.

This is one of the few randomised controlled studies that has evaluated a strategy to reduce burnout in junior doctors. ${ }^{29}$ The use of a validated outcome tool, specifically the MBI, was considered a strength of this study. Although a negative study, the findings contribute to the overall literature on junior doctor burnout.

There are a number of limitations recognised. The study was likely underpowered to detect a difference in burnout following debriefing. The use of other tools to measure burnout and stress such as the Professional Quality of Life and the Copenhagen Burnout Inventory may have produced different results.

The sample was largely representative of those interns who were available to participate. However, there is potential for selection bias with those who did not elect to participate, as they may have been less affected by burnout, or, conversely, unable to participate despite experiencing burnout. Attendance at the debriefing sessions was not always $100 \%$, leading to the potential argument that those who were regularly in attendance were experiencing more or less stress. On an organisational level, all supervisors of interns need to support the attendance of junior doctors at debriefing sessions. The debriefing sessions were designed to allow flexibility based on the agenda set by the participants: this may make the debriefing process hard to replicate, but contextually more valid.

The differences observed in women could be a result of type 1 error due to the statistical tests comparing a number of baseline variables. This could be recognised as a limitation.

This study was conducted in an inner city teaching hospital in Sydney, Australia. The findings may not be applicable to other international settings with different work practices. The results

Table 6 Change in burnout scores post intervention

\begin{tabular}{lrrr}
\hline & $\begin{array}{l}\text { Control mean } \\
\text { difference } \\
(95 \% \mathrm{Cl})\end{array}$ & $\begin{array}{l}\text { Intervention } \\
\text { mean difference } \\
\text { (95\% Cl) }\end{array}$ & $\begin{array}{l}\text { Interaction } \\
\mathbf{p} \text { value }\end{array}$ \\
\hline Emotional exhaustion score & $0.4(-2.8$ to 3.5$)$ & $-0.3(-5.3$ to 4.8$)$ & 0.87 \\
Cynicism score & $-0.2(-2.3$ to 1.9$)$ & $0.1(-3.7$ to 3.9$)$ & 0.91 \\
Professional efficacy score & $1.6(-1.2$ to 4.4$)$ & $-0.6(-3.6$ to 2.5$)$ & 0.33 \\
Total score & $-1.4(-7.2$ to 4.4$)$ & $0.4(-5.7$ to 6.5$)$ & 0.83 \\
\hline
\end{tabular}

however are likely to reflect the practice in other Australian hospitals, and further data collection surrounding current rates of burnout within this setting is encouraged.

This pilot study could be expanded to involve multiple hospital sites to maximise sample size and debriefing sessions extended for a whole year with pre and post outcomes being measured at initiation and completion of the intern year.

In conclusion, burnout is a problem that affects junior doctors and adversely impacts on the quality and safety of patient care. Few interventions are currently available. The results of this study demonstrate that further investigation with debriefing sessions has merit. From our research, it appears that junior doctors derive some satisfaction from these sessions and a larger multicentre study is required to assess whether debriefing should be implemented on a wider scale. Based on this feasibility study we believe this strategy could be easily implemented in hospitals around Australia and internationally.

\section{Box 3 Quotes supporting identified stressors}

1. It's more not really being sure where I'm headed next and feeling like this job is only going to last to the next year then I'm going to have to reapply for something. I'm not quite sure what I want.

2. Knowing that there's so many new interns coming out and so there's this pressure to get on to a training program and feel like you're in the system.

3. I was involved in a traumatic arrest early on in internship and it took me days to recover. (To work out what went wrong, could I have done anything?) It was unfortunate that my registrar was busy and she just left me there and I had to tell the family and all that sort of thing.

\section{Box 4 Quotes supporting coping mechanisms}

1. One of the best things was talking to other people and finding out that you're not alone, that everyone has similar experiences

2. Everyone goes to the pub on a Friday or you go out to dinner with your friends you seem to talk about work a lot and I think that helps. You talk about scenarios that you found difficult or things that you found interesting and even though it is talking shop it kind of allows you to debrief a bit.

3. It's not so much the booze (alcohol) but it's what it represents - being home, being relaxed, getting changed and letting your hair down. That was something that drove me to a degree-something to look forward to at the end of the day. 


\section{Box 5 Opinions on utility of debriefing sessions}

1. [I was] able to see that other interns who appeared to be holding it together better were having the same issues [as me] and learning how they dealt with those situations which I felt I could not manage [helped]

2. [it was] Good to hear that other people were experiencing similar issues at work-[I was] not alone.

3. Voluntary, regular sessions so interns could drop in when they feel they need to (and are not made to feel bad by interns who have no issues to talk through/no problems at work)

\section{Main messages}

- Burnout can directly affect the junior doctors and potentially patient care.

- Burnout affected up to $68 \%$ of doctors in their 1 st year of internship with higher representation in women than men.

- An intervention, in the form of debriefing sessions, aimed to reduce the prevalence of burnout, and was well received by the cohort, with $90 \%$ concluding that the intervention was a source of emotional and social support for them.

\section{Current research questions}

- What are future strategies that can reduce junior doctor burnout and improve well-being?

- The feasibility of instituting debriefing programmes on a larger scale, nationally and potentially internationally?

- Are there new and emerging sources of stress and burnout in the junior doctor given the changing climate of the work force?

\section{Key references}

- AMA. AMA Survey Report on Junior Doctor Health and Wellbeing. 2008

- Prins J, Gazendam-Donofrio S, Tubben B, et al. Burnout in medical residents: a review. Med Educ 2007;41:788-800.

- Thomas NK. Resident Burnout. JAMA 2004;292:2880-9.

Contributors All five authors listed have contributed equally to the conception and design of this study as well as data collection, analysis and interpretation. There has been equal involvement in the writing of this article and final approval.

Competing interests None declared.

Ethics approval Royal Prince Alfred Human Research and Ethics Committee (Ethics Approval X11-0084).

Provenance and peer review Not commissioned; externally peer reviewed.

\section{REFERENCES}

1 Henning M, Hawken S, Hill A. The quality of life of New Zealand doctors and medical students: what can be done to avoid burnout? $N$ Z Med J 2009;122:102-10.

2 AMA. AMA Survey Report on Junior Doctor Health and Wellbeing. 2008.

3 Shanafelt T, Bradley K, Wipf J, et al. Burnout and self-reported patient care in an internal medicine residency program. Ann Intern Med 2002;136:358-67.

4 Willcock S, Daley M, Tennant C, et al. Burnout and psychiatric morbidity in new medical graduates. Med J Aust 2004;181:357-60.

5 Campbell J, Prochazka A, Yamashita T, et al. Predictors of persistent burnout in internal medicine residents: a prospective cohort study. Acad Med 2010;85:1630-4

6 Uallachain G. Attitudes towards self-health care: a survey of GP trainees. Ir Med J 2007;100:489-91

7 West CP, Tan AD, Shanafelt TD. Association of resident fatigue and distress with occupational blood and body fluid exposures and motor vehicle incidents. Mayo Clin Proc 2012;87:1138-44.

8 Fahrenkopf A, Sectish T, Barger L, et al. Rates of medication errors among depressed and burn out residents: prospective cohort study. BMJ 2008;336:488-91.

9 Feld J, Heyse-Moore L. An evaluation of a support group for junior doctors working in palliative medicine. Am J Hosp Palliat Care 2006;23:287-96.

10 Markwell A, Wainer Z. The health and wellbeing of junior doctors: insights from a national survey. Med J Aust 2009;191:441-4.

11 Marine A, Ruotsalainen J, Serra C, et al. Preventing occupational stress in healthcare workers. Cochrane Database Syst Rev 2006;(4):CD002892.

12 Prins J, Gazendam-Donofrio S, Tubben B, et al. Burnout in medical residents: a review. Med Educ 2007:41:788-800.

13 Hochberg $M$, Berman $R$, Kalet $A$, et al. The stress of residency: recognizing the signs of depression and suicide in you and your fellow residents. Am J Surg 2013;205:141-6.

14 Bair J, Greenspan B. TEAMS: teamwork training for interns, residents, and nurses Hosp Community Psychiatry 1986:37:633-5.

15 Shapiro S, GE S, Bonner G. Effects of mindfulness-based stress reduction on medica and premedical students. J Behav Med 1998;21:581-99.

16 Ospina-Kammerer V, Figley C. An evaluation of the respiratory one method (ROM) in reducing emotional exhaustion among family physician residents. Int J Emerg Ment Health 2003;5:29-32.

17 Dabrow S, Russell S, Ackley K, et al. Combating the stress of residency: one school's approach. Acad Med 2006:81:436-9.

18 Dyrbye LN, Thomas MR, Huschka MM, et al. A multicenter study of burnout, depression, and quality of life in minority and nonminority US medical students. Mayo Clin Proc 2006;81:1435-42.

19 Michie S, Williams S. Reducing work related psychological ill health and sickness absence: a systematic literature review. Occup Environ Med 2003;60:3-9.

20 Freudenberger H. Staff burnout-out. J Soc Issues 1974;30:159-65.

21 Maslach C, Goldberg J. Prevention of burnout: new perspectives. App/ Prev Psychol 1998; 7:63-74.

22 Schenarts PJ. Debriefing is an effective method for providing feedback and ensuring adherence to best clinical practice by residents in the intensive care unit. Crit Care Med 2007;35:957-8.

23 Maslach C, Jackson SE, Leiter MP, et al. Maslach Burnout Inventory Instruments and Scoring Guides. Forms: General, Human Services, \& Educators. Palo Alto: Consulting Psychologist Press, 1986.

24 Rafferty J, Lemkau J, Purdy R, et al. Validity of the Maslach Burnout Inventory for family practice physicians. J Clin Psycho 1986;42:488-92.

25 Schaufeli W. Stress in health professionals: psychological and organizational causes and interventions. In: Firth-Cozens J, Payne R. eds. Burnout. Chichester, NY: Wiley, 1999.

26 Taris TW, Schreurs PJG, Schaufeli WB. Construct validity of the Maslach Burnout Inventory-General Survey: a two sample examination of its factor structure and correlates. Work and Stress 1999:13:233-7.

27 Thomas NK. Resident Burnout. JAMA 2004;292:2880-9.

28 West CP, Shanafelt TD, Kolars JC. Quality of life, burnout, educational debt, and medical knowledge among internal medicine residents. JAMA 2011;306:952-60

29 McCray LW, Cronholm PF, Bogner HR, et al. Resident physician burnout: is there hope? Fam Med 2008;40:626-32. 